\title{
CIRCUMNUCLEAR STRUCTURES IN THE INTERACTING SEYFERT GALAXY NGC 1241: KINEMATICS AND OPTICAL/INFRARED MORPHOLOGY
}

\author{
Rubén J. Díaz, ${ }^{1,2}$ Horacio Dottori, ${ }^{3}$ Nelson Vera-Villamizar, ${ }^{4}$ and Gustavo Carranza ${ }^{1,5}$ \\ Received 2002 November 4; accepted 2003 July 16
}

\begin{abstract}
We studied the spiral pattern in the inner 6."5 $\left(1^{\prime \prime}=257 \mathrm{pc}\right)$ central regions of the interacting active nucleus galaxy NGC 1241 using Gemini North Telescope high-resolution $K_{s^{-}}$and $J$-band images and Hubble Space Telescope (HST) Pa $\alpha$ and $H$ - and $(V+R)$-band images with high resolution in the range from $\sim 0$ ". 1 to $\sim 0$ ".3 along with intermediate to large-scale spectroscopy using the Multifunction Spectrograph at the Córdoba Observatory in Argentina. Our analysis of $\mathrm{Pa} \alpha$ emission images revealed a faint two-armed leading spiral pattern ending in the 5". $6 \times 3$ ".4 clumpy ring discovered by Böker and coworkers, harboring a 1".6 long barlike structure almost perpendicular to the large-scale bar of NGC 1241. When we applied two-dimensional Fourier analysis at circumnuclear scales, we found that a two-arm trailing mode was dominant in $K_{s}$ - and $J$-band images while the $(V+R)$-band images showed more complex structural features with a strong onearmed trailing mode. One-dimensional Fourier analysis showed a corotation (CR) located outward from the edge of the Pa $\alpha$ bar. Our kinematics data gave an angular speed $\Omega_{\mathrm{CN}}$ of $350 \pm 50 \mathrm{~km} \mathrm{~s}^{-1} \mathrm{kpc}^{-1}$ for the trailing mode pattern. The rotation curve showed that the circumnuclear ring is located just inside the large-scale pattern inner Lindblad resonance (ILR), which has a radius of about $r \sim 1 \mathrm{kpc}$. We also found, within the uncertainties present in such measurements, that the outer Lindblad resonance (OLR) of the circumnuclear pattern is coincident with the large-scale pattern ILR, indicating a possible connection between circumnuclear and global dynamics. Nevertheless, the estimated high molecular gas fraction $(\geq 13 \%)$ and the inner pattern high angular speed at the central region of NGC 1241 point to a nuclear bar formation via self-gravitational instability.
\end{abstract}

Subject headings: galaxies: active — galaxies: individual (NGC 1241) — galaxies: kinematics and dynamics — galaxies: spiral — galaxies: structure methods: numerical — techniques: spectroscopic

\section{INTRODUCTION}

The connection between nuclear activity and star formation (Terlevich \& Melnick 1985) has received growing attention over the last few years (Schmitt, Storchi-Bergmann, \& Fernandes 1999; Ivanov et al. 2000), with nuclear bars being invoked as a possible mechanism for fueling not only nuclear activity but also circumnuclear star formation (Heller \& Shlosman 1994; Shlosman, Frank, \& Begelman 1989; Maiolino et al. 2000). Several of the galaxies with hot spots are composite in nature with a central LINER or Seyfert-like nucleus surrounded by star-forming rings (Pastoriza et al. 1993), the relationship between these rings of star formation and resonances that help to accumulate gas in these zones having been extensively discussed in the literature (Dottori 1990; Piner, Stone, \& Teuben 1995; Buta \& Combes 1996; Buta et al. 2000).

The main obstacle to studying perturbations of the innermost circumnuclear disks (Knapen et al. 1995; Maiolino et al. 2000) has been the lack of images with sufficiently high spatial resolution. Dust is a common feature of the

\footnotetext{
1 Observatorio Astronomico, Universidad Nacional de Córdoba, Laprida 854, 5000 Córdoba, Argentina.

${ }^{2}$ Secretaria de Ciencia y Tecnologia, Universidad Nacional de Córdoba, Cordoba, Argentina.

${ }^{3}$ Instituto de Fisica, Universidade Federal do Rio Grande do Sul, CEP RS 91501-970 Porto Alegre, Brazil.

${ }^{4}$ Instituto Nacional de Astrofisica, Óptica y Electrónica, Apartado de Correo 51 y 216, 72000 Puebla, Mexico.

${ }^{5}$ Consejo Nacional de Investigaciones Cientificas y Tecnicas (CONICET), Rivadavia 1917, Buenos Aires 1033, Argentina.
}

circumnuclear environment of Seyfert galaxies (Regan \& Mulchaey 1999; Martini \& Pogge 1999), the dust often being confined to the disk where it causes significant extinction, which contributes to reduced resolution. Observations at near-infrared (NIR) bands are very important because they allow penetration of the dust and are better able to detect perturbations in the original old stellar disk population, such perturbations being the possible missing link between circumnuclear star-forming rings and nuclear activity (Knapen et al. 1995). Block \& Puerari (1999) have shown that spiral arm structure appears much more simple in NIR $K_{s}$-band images than in visual band images. Contrary to expectations, no correlation has been found between the pitch angle of galaxy arms measured from $K_{s}$-band images and Hubble stellar classification using visual bands (Block \& Puerari 1999; Seigar \& James 1998), and it is therefore reasonable to assume that there would be differences between NIR and visual band images of the circumnuclear spiral structure discussed in this paper.

The SB(s)b galaxy NGC 1241 (Fig. 1) harbors a Seyfert 2 nucleus with a circumnuclear ring of star formation recently detected in $\mathrm{Pa} \alpha$ (Böker et al. 1999). In this paper we discuss a possible connection between disk perturbations of a few hundred parsecs at the center of NGC 1241 and the $11 \mathrm{kpc}$ long $(h=0.75)$ main bar. We used the high-resolution $\sim 0$ "!3 $J$ - and $K_{s}$-band images from the Gemini North Telescope and Hubble Space Telescope (HST) $\sim 0^{\prime \prime} 1$ resolution $H$ - and $\sim 0$ ".2 $(V+R)$ - and $\mathrm{Pa} \alpha$-band archival images along with intermediate to large-scale spectroscopy obtained using equipment at Córdoba Observatory in Argentina. 


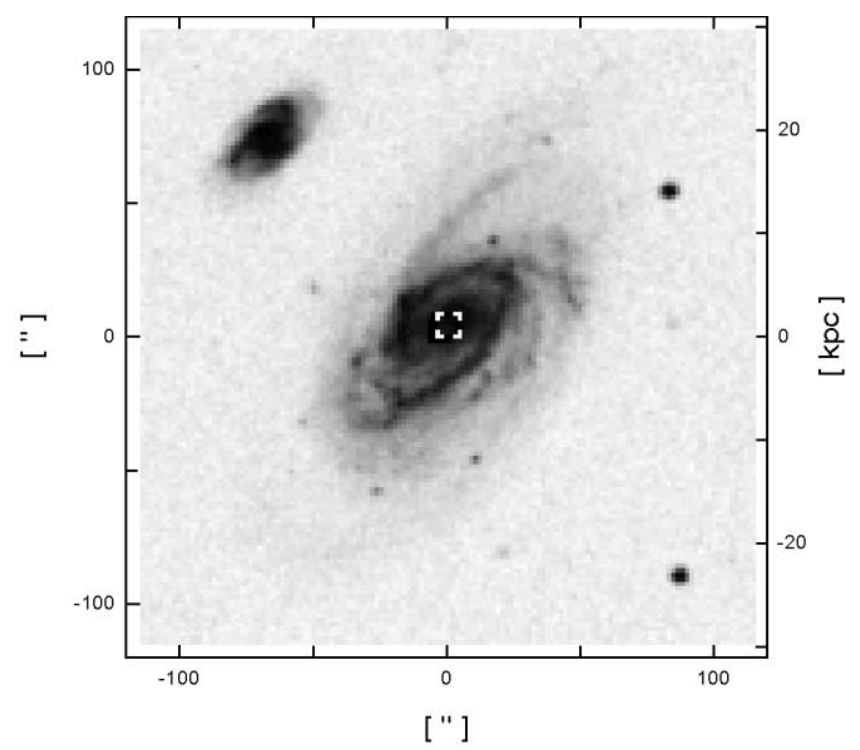

FIG. 1.-DSS field of the Seyfert galaxy NGC 1241 and its companion NGC 1242. The box corresponds to the inner $2 \mathrm{kpc}$ of NGC 1241 (Fig. 2). North is at the top and east is to the left.

In $\S 2$ we describe our methods of observations and data reduction, while in $\S \S 3$ and 4 we reveal the innermost spiral structure using the well-tested Image Reduction and Analysis Facility (IRAF) package and two- and one-dimensional fast Fourier transform (FFT) to analyze the innermost perturbation and detect corotation (CR), explore the links between the global and circumnuclear kinematics of NGC 1241 , and discuss the inner pattern origin and age.

\section{OBSERVATIONS AND DATA REDUCTION}

\subsection{Imaging}

On 2000 September 30 we used the Quick Start service of the Gemini North $8.1 \mathrm{~m}$ telescope for NIR imaging using Hokupa's natural guide star, curvature-sensing adaptive optics system, to feed the dedicated Quick NIR camera (QUIRC) fitted with a $1024 \times 1024 \mathrm{HgCdTe}$ array sensitive to $1-2.5 \mu$ m radiation to give a final scale of 0.0197 pixel $^{-1}$. We also used NIR and broad optical band archival HST images, the NIR images having been taken with the infrared camera NIC3 $\left(0\right.$ ".20 pixel $\left.^{-1}\right)$ and either the F160W band continuum filter or the $\mathrm{F} 190 \mathrm{~N}$ redshifted $\mathrm{Pa} \alpha(1.90 \mu \mathrm{m})$. Broadband optical images were taken with the Wide Field Planetary Camera 2 (WFPC2) (0".046 pixel $\left.^{-1}\right)$ and the F606W visible plus red $(V+R)$ filter.

Standard data reduction procedures were applied to images, which were all rotated to the standard orientation of north to the top and east to the left. The achieved FWHM of the Gemini+Hokupa'a optic system was $\approx 0$ ". 4 in $J$ band and $\approx 0.3$ in $K_{s}$ band (Strehl ratio was only $\approx 0.02$ ) measured on the point-spread function (PSF) of a field star and estimated on the target galaxy. Image deconvolution was not applied at this stage because image quality was good enough to be compared with HST NIC3 data. The FWHM spatial resolution of the HST WFPC2 images was 0.11 (25.7 pc; $h=0.75)$ and that of NIC3 0.3 as measured from unresolved sources in the frames.
We applied the IRAF ELLIPSE program to $K_{s}, J$, and $(V+R)$ images and produced densely nested twoparameter constrained isophotes that made the spiral nature of the structures more evident (Figs. $2 d-2 f$ ). We then applied one- and two-dimensional FFT to analyze the innermost perturbations and to detect CR and explored the links between the circumnuclear and global kinematics of this galaxy.

\subsection{Spectroscopy}

In 2000 January and 2001 January we observed NGC 1241 spectroscopically using the Córdoba Observatory 1.54 $\mathrm{m}$ reflector fitted with the Multifunctional Integral Field Spectrograph (for a brief description see Díaz et al. 1999) in long-slit and direct imaging modes, using a Tektronics $1024 \times 1025$ charge-coupled device (CCD) and a 1200 line $\mathrm{mm}^{-1}$ grating in order 1 , at wavelengths $6200-7000 \AA$. Almost $10 \mathrm{hr}$ of $1.5 \AA$ resolution spectra were obtained, mostly covering the central region of the galaxy, at four different slit positions, with zero-order imaging allowing us to register slit positions with high accuracy. Standard data reduction procedures were applied to the spectra, which were rebinned to $0.5 \mathrm{pixel}^{-1}$. The slice width of the zones of highest signal-to-noise ratio $(\mathrm{S} / \mathrm{N})$ was 0 ." 5 , and outside the circumnuclear regions the slice width was progressively widened, up to $15^{\prime \prime}$ in the outermost points. Angular resolution was close to 1 '! 5 , so the radial velocity field was slightly oversampled in the nuclear region. After wavelength calibration, the instrumental width of the night-sky emission lines was $\sim 90 \mathrm{~km} \mathrm{~s}^{-1}$.

Radial velocities were derived by measuring the centroids of Gaussian curves fitted at the profiles of the strongest emission lines $(\mathrm{H} \alpha$, [N II] $\lambda \lambda 6548,6584$, and [S II] $\lambda \lambda 6717$, 6731 ), but for lower $\mathrm{S} / \mathrm{N}$ we only fitted the $\mathrm{H} \alpha$ emission line. Radial velocities were determined up to $\sim 50^{\prime \prime}$ from the nucleus $(\sim 13 \mathrm{kpc})$. Since most of the radial velocities where measured from one emission line, we used the procedure described in Díaz et al. (2000) to determine the uncertainty of each velocity value. From symmetry considerations in the central portion of the NGC 1241 rotation curve, we derived a heliocentric systemic velocity of $V_{\text {sys }}=4016 \pm 10$ $\mathrm{km} \mathrm{s}^{-1}$, consistent with the value given by Persic \& Salucci (1995), from a less resolved rotation curve.

\section{DATA ANALYSIS AND RESULTS}

\subsection{The Morphology of the Central Kiloparsec Region}

The $J$ and $K_{s}$ images of Figure 2 show a smooth, inclined disk with approximately elliptical isophotes of varying position angle. The ratio of major to minor axis of the outermost isophotes in the $J, K_{s}$, and $(V+R)$ images reveals a disk inclination of $52^{\circ}$, consistent with the value given by Tully (1988) for the large-scale disk.

The $(V+R)$ image shows an asymmetrical arm and diverse secondary features that suggest a more complex arm pattern than can be seen in the NIR images. The five central $(V+R)$ isophotes of Figure $2 d$ show a weak centered barlike feature connected to the arms, this bar being oriented approximately northwest-southeast and with a length comparable to the $\mathrm{Pa} \alpha$ bar. By smoothing the $K_{s}$-band ellipses map (Fig. $2 f$ ), we were able to detach the dominant twoarm structure shown in Figure 3 where it is compared with the $\mathrm{Pa} \alpha$ image. A mathematically rigorous analysis of the 


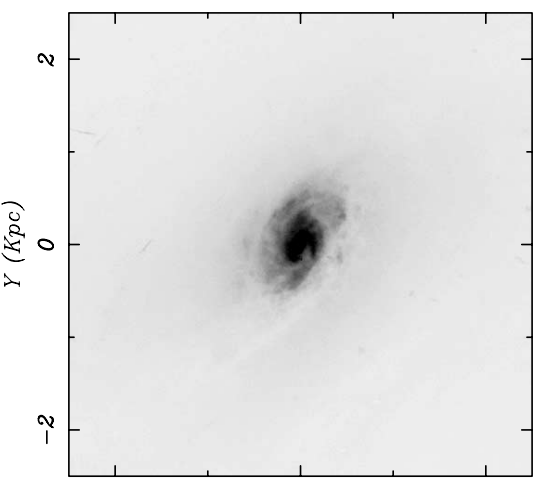

FIG. $2 a$

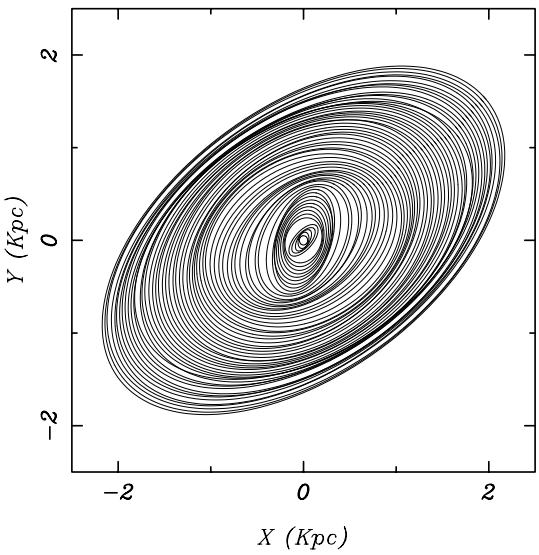

Fig. $2 d$

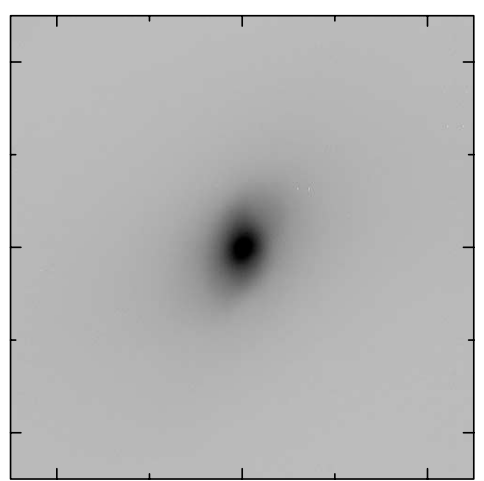

FIG. $2 b$

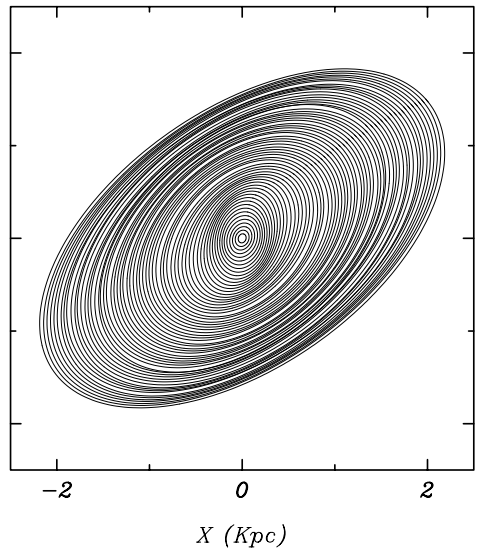

FIG. $2 e$

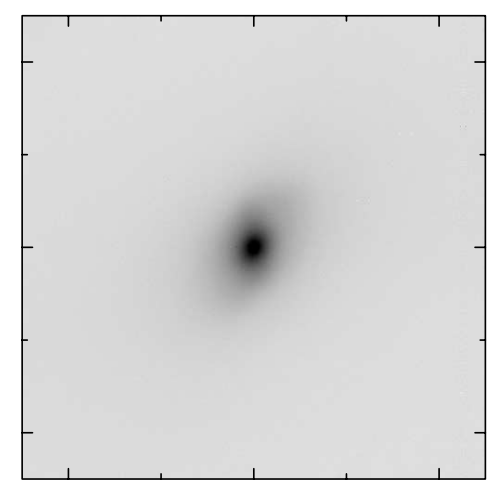

FIG. $2 c$

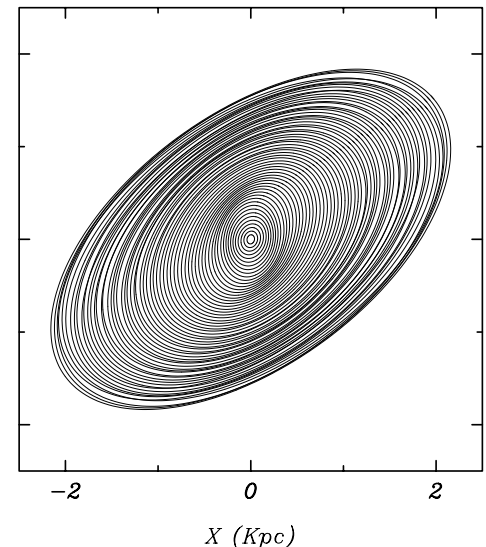

FIG. $2 f$

FIG. 2.- (a) HST optical and $(b, c)$ Gemini IR images of NGC 1241 and $(d-f)$ the corresponding fitted ellipses. North is at the top and east is to the left.

inner arm pattern based on two-dimensional FFT was carried out, the results being given in the table in $\S 3.2$.

The $\mathrm{Pa} \alpha$ image (Fig. 3) shows a well-defined emitting ring (Böker et al. 1999), with a brightness radial profile peak at a radius $r=710 \pm 80 \mathrm{pc}$, and a small central barlike oriented north-south feature (P.A. $=10^{\circ} \pm 5^{\circ}$ ), with an on-the-sky projected length of $410 \pm 50 \mathrm{pc}$. This barlike feature, almost perpendicular to the main bar, has no strong dust lanes, which explains why it was not detected in the highresolution color maps of active galactic nuclei (AGNs)

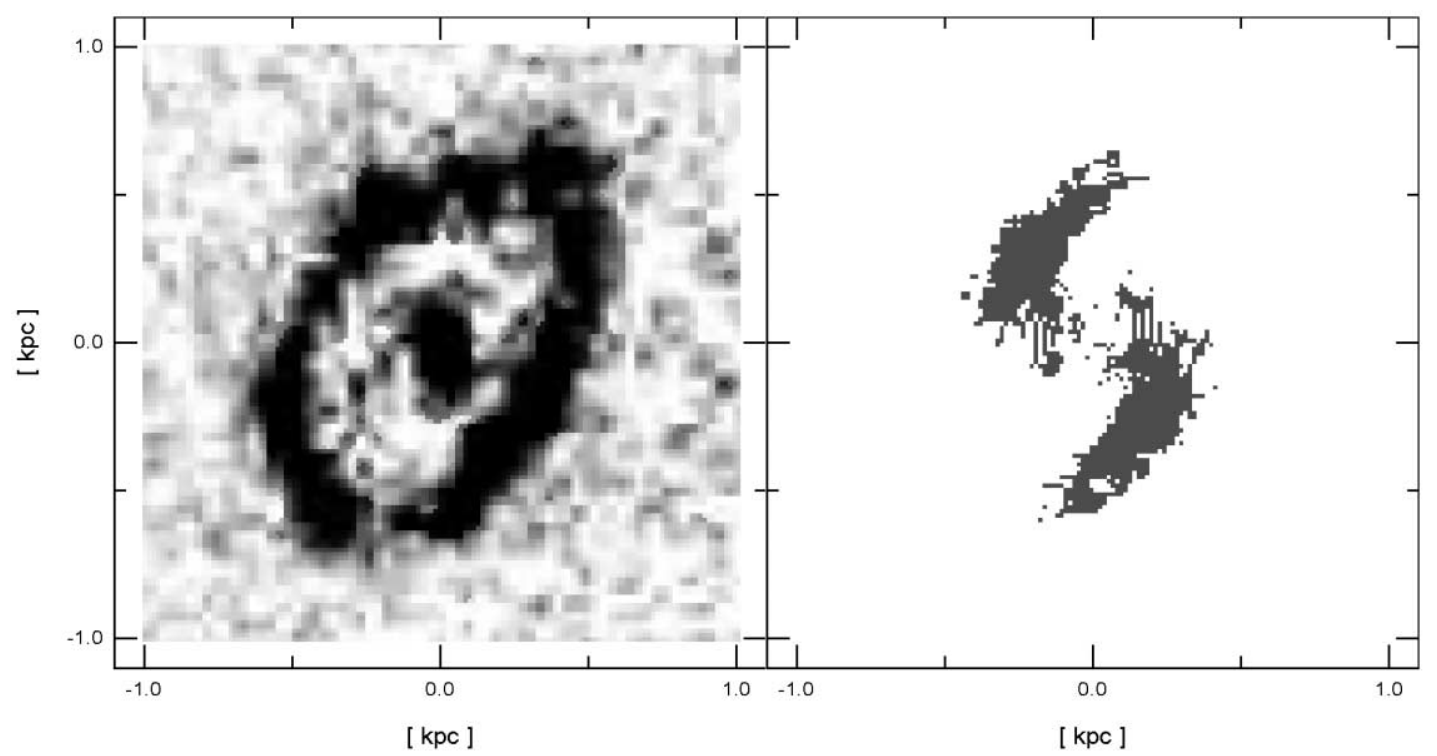

FIG. 3.-Pa $\alpha$ emission ( $H$-band continuum subtracted) in the central $2 \mathrm{kpc}$ of NGC 1241 . Note the barlike central structure and the faint leading spiral in $\mathrm{Pa} \alpha$ emission. The right-hand panel shows the $K_{s}$-band $m=2$ component, obtained from Fig. $2 f$. The $m=2$ mode completely dominates the $K_{s}$-band two-dimensional Fourier spectrum (Fig. 5). North is at the top and east is to the left. 


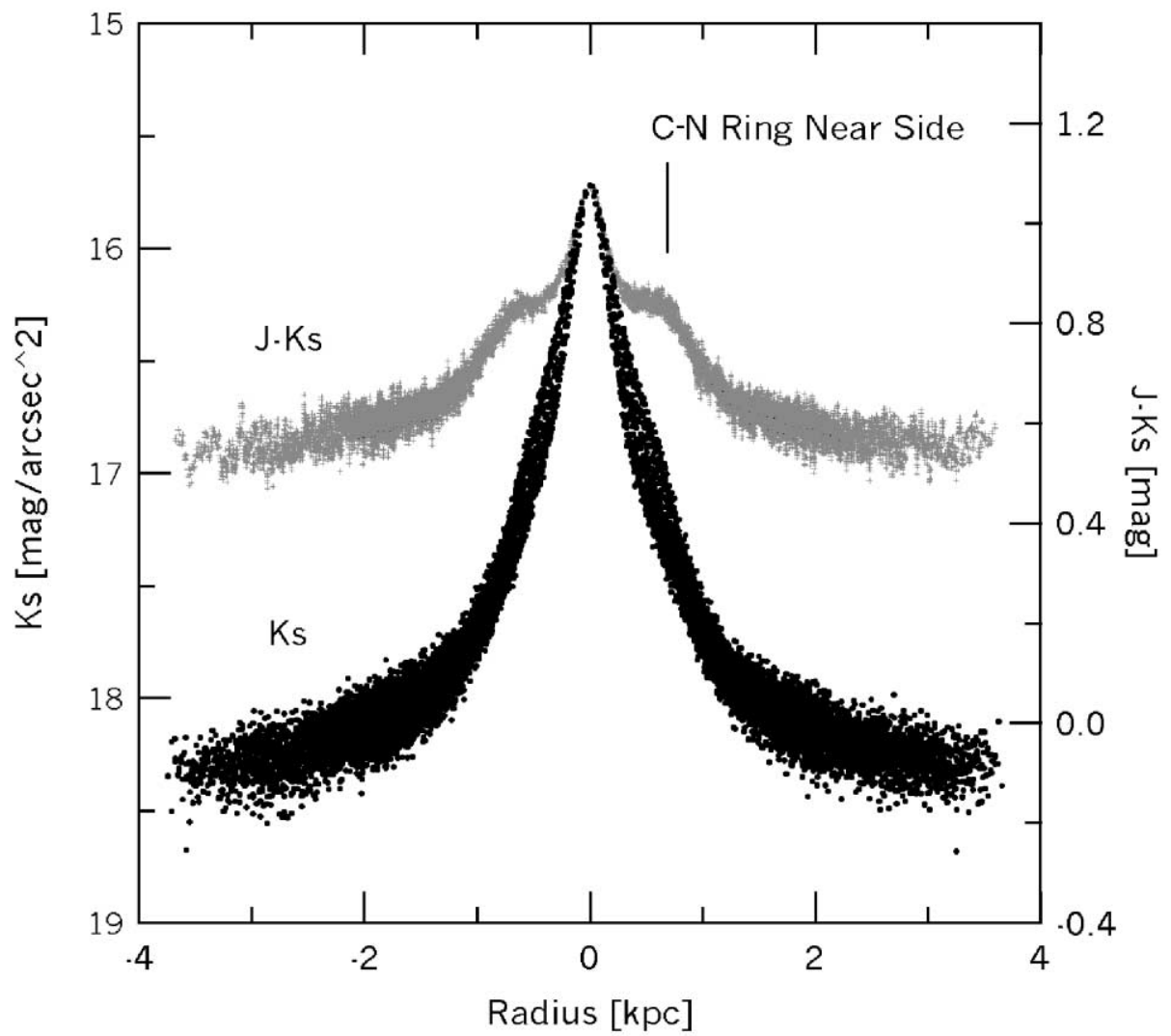

Fig. 4. $-K_{s}$ and $\left(J-K_{s}\right)$ photometric values for rebinned pixels of 0 ". 1 vs. deprojected radius. The pixels to the northeast are separated from those to the southwest of the major axis (positive radii). Note the increasing reddening inward and the remarkably similar radial behavior on both sides of the line of nodes.

produced by Regan \& Mulchaey (1999). A faintly emitting two-armed counterclockwise spiral structure can also be seen linking the extremes of the Pa $\alpha$ bar to the ring. As compared to the $\mathrm{Pa} \alpha$ small bar, the nuclear bar in $J$ and $K_{s}$ images is $\approx 1.5$ times larger and rotated counterclockwise by $\approx 25^{\circ}$. The $K_{s}$-band arms smoothly fade on the star-forming ring and disappear outward. Since the $K_{s}$ - and $J$-band spiral structures are morphologically similar in this paper, we will only discuss the $\mathrm{Pa} \alpha,(V+R)$, and $K_{s}$ spiral patterns.

The $K_{s}$ band suffers much less extinction than that seen in optical bands, and our $K_{s}$-band images do not show any of the dust+hot-spot features seen in the $(V+R)$-band images. As pointed out by Martini \& Pogge (1999), the effect of dust cannot be easily quantified because it is to be expected that this component is well mixed with the stars. To ascertain whether or not the morphology seen in $K_{s}$ band images is differentially affected by the presence of dust, we carried out pixel photometry, plotted $K_{s}$ and $\left(J-K_{s}\right)$ versus deprojected radius, and separated all the pixels to the northeast from those to the southwest of the major axis (Fig. 4). This figure shows increasing reddening inward with a remarkably similar radial behavior on both sides of the line of nodes, as well as a plateau delimited by the $\mathrm{Pa} \alpha$ ring with $\left(J-K_{s}\right) \sim 0.85$ at the edge and about 1.05 mag at the nucleus, the region inside the ring presenting the same color trend. The effect of dust on the $K_{s}$ band is azimuthally uniform and does not show any abnormal distribution that could differentially affect the $\mathrm{Pa} \alpha$ and $K_{s}$ morphology. The southwest wing of the $\left(J-K_{s}\right)$ plateau is slightly redder, something that has previously been pointed out by Regan \&
Mulchaey (1999), who also claim that the color excess is $(V-H) \sim 1.1$ at the near side of the ring. The main morphological characteristics discovered by us are summarized in Table 1.

\subsection{Inner Arms Pattern}

In order to mathematically characterize the perturbations in the central kiloparsec, we carried out a two-dimensional FFT main component analysis (Kalnajs 1975; Considère \& Athanassoula 1982; Puerari \& Dottori 1992) of the inner arm pattern using the procedure outlined by VeraVillamizar et al. (2001), which consists of enhancing the contrast of the arms by subtracting an exponential disk and decomposing the spiral arms into six logarithmic spirals that in polar coordinates $(r, \theta)$ have the form $r=r_{0} \exp [(-m /$ $P) \theta$, where $m$ equals the number of arms and $P$ is related to the spiral pitch angle $p$ of the spiral by the formula $p=\tan ^{-1}(-m / P)$. This algorithm gives the amplitude of each Fourier $A(p, m)$ component as a function of the deprojected distribution of light $I(r, \theta)$ and also the Fourier radial density function $S_{m}(r)$ of the $m$ component, which indicates the importance of that particular component at a given radius. Table 2 gives the Fourier parameters, pitch angle, and arm length for the dominant modes, while Figure 5 shows the most prominent $(V+R)_{-}, K_{s^{-}}$, and $\mathrm{Pa} \alpha$-band Fourier $A(p, m)$ spectra.

The $(V+R)$ complex arm structure mentioned in the previous section is reflected in the richness of the Fourier spectrum, which has a strong $m=1$ mode and two $m=2$ 
TABLE 1

The Main Structures of NGC 1241

\begin{tabular}{|c|c|c|c|c|c|c|}
\hline Feature & $\begin{array}{l}\text { P.A. } \\
\text { (deg) }\end{array}$ & $\begin{array}{l}\text { Axial Ratio } \\
\quad d / D\end{array}$ & $\begin{array}{c}i \\
(\mathrm{deg})\end{array}$ & $\begin{array}{l}\text { Major-Axis Length } \\
\quad(\operatorname{arcsec})\end{array}$ & $\begin{array}{l}\text { Major-Axis Length } \\
\qquad(\mathrm{kpc})\end{array}$ & $\begin{array}{c}\text { Radius } \\
(\mathrm{kpc})\end{array}$ \\
\hline NGC 1242 (disk) ....................... & 45 & 0.46 & $63 \pm 5$ & $50 \pm 3$ & $12.8 \pm 0.8$ & $24.7^{\mathrm{a}}$ \\
\hline 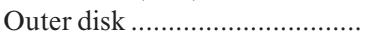 & 151 & $0.64 \pm 0.02$ & $52 \pm 3$ & $220 \pm 20^{b}$ & $55 \pm 5$ & 22.5 \\
\hline 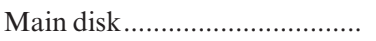 & 140 & 0.59 & 54 & $\sim 100$ & 25.7 & $5.1^{\mathrm{c}}$ \\
\hline Main bar........................................ & 94 & $0.29 \pm 0.03$ & $\ldots$ & $36 \pm 4$ & $10.5 \pm 1.0^{\mathrm{d}}$ & $5.3^{\mathrm{d}}$ \\
\hline 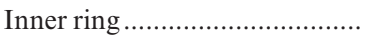 & 140 & $0.60 \pm 0.05$ & $53 \pm 4$ & $\sim 19$ & $\sim 9.8$ & 4.9 \\
\hline Bulge & $\ldots$ & $\ldots$ & $\ldots$ & $9.5 \pm 1.5^{\mathrm{e}}$ & $\ldots$ & $2.4^{\mathrm{e}}$ \\
\hline 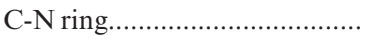 & $150 \pm 3$ & $0.61 \pm 0.03$ & $53 \pm 3$ & $5.5 \pm 0.4$ & $1.4 \pm 0.1$ & 0.71 \\
\hline $\mathrm{C}-\mathrm{N}$ bar (ionized gas) .................. & $10 \pm 5$ & $0.4 \pm 0.1$ & $\ldots$ & $1.6 \pm 0.2^{\mathrm{f}}$ & $0.51 \pm 0.05^{\mathrm{d}}$ & $0.26^{\mathrm{d}}$ \\
\hline $\mathrm{C}-\mathrm{N}$ bar (stars) .......................... & $110 \pm 20$ & $\ldots$ & $\ldots$ & $\sim 2.4^{\mathrm{g}}$ & $\sim 0.7^{\mathrm{d}}$ & $0.35^{\mathrm{d}}$ \\
\hline
\end{tabular}

a Projected P.A. and distance to NGC 1241 nucleus.

b Optical diameter at the sky level of the DSS red image.

${ }^{\mathrm{c}}$ Exponential scale length and ellipticity from Cunow 2001. We estimated the major-axis length of the main disk, bar, and inner ring by the slope changes of the azimuthal profiles at the DSS and WFPC2 images.

d Projected value on the galaxy main disk.

e $\mathrm{R}^{1 / 4}$ scale length in the $K_{\mathrm{s}}$-band images, fitted with a main disk with exponential scale length of $20^{\prime \prime}$.

${ }^{\mathrm{f}}$ Estimated from the isophote maps of the $\mathrm{Pa} \alpha$ emission and also derived from Fourier spectra.

g Estimated from the isophote maps in $J, H$, and $K_{s}$ bands and also derived from Fourier spectra.

modes, a bar and a trailing arm. Other less meaningful components are not given in Table 2 because they serve only to describe the patchy nature of the spirals and the influence of the $(V+R)$-band extinction. As mentioned in $\S 3.1$, the $K_{s}$-band spiral pattern is smooth, and it is very well represented by a pure $m=2$ mode. As generally accepted, we assume that the $K_{s}$ band shows the effect of perturbations on the disk old stellar population (Block et al. 1994; Block \& Puerari 1999), a previous study of Schweizer (1976) having shown that NIR colors are influenced in $80 \%$ or more by disk old stars.

In $\operatorname{Pa} \alpha, m=2$ mode reveals the small central barlike structure and the two leading arms extending from the bar (Fig. 3). The emitting barlike structure might be the result of the juxtaposition of two or more $\mathrm{H}$ II regions, but the resolution of the image is not sufficient to spatially resolve them. Nevertheless, as will be discussed in $\S 3.3$, the onedimensional Fourier transform also reveals the existence of both the barlike structure and the leading pattern. The Fourier spectrum also shows that the main $m=2$ mode is modulated through less important odd modes, which we interpret as representing the ring clumpiness.

It is important to point out that the $K_{s}$ image shown in Figure 3 demonstrates that the armlike structure is located inside the $\mathrm{Pa} \alpha$ star-forming ring, indicating the behavior of perturbations over a hundreds of parsec scale. The NIR cir- cumnuclear perturbation properties revealed by our analysis resemble those found in galaxy M100 by Knapen et al. (1995).

\subsection{Circumnuclear Disk Corotation Resonance}

The identification of resonances in spiral galaxies is a matter of increasing interest for spiral arm theory and modeling (Seigar \& James 1998; Lindblad, Lindblad, \& Athanassoula 1996). Photometric methods have been extensively used to detect resonances, mainly as a result of the fact that these methods consume less observation time compared to spectroscopic methods. Elmegreen, Elmegreen, \& Montenegro (1992) pointed out that evidence for CR resonances is clearly visible in gas-rich galaxies. According to Schweizer (1976; see also Beckman \& Cepa 1990), a comoving observer sited at the $\mathrm{CR}$ and looking outward and inward would see the shock front change from one side of the spiral to the other (the so-called shock front jump), reversing the order in which the younger and older disk stellar populations appear in azimuthal profiles across the arms. Puerari \& Dottori (1997) proposed that the shock front jump could be detected by analyzing the relative behavior of the spiral density wave phase $\Theta_{\mathrm{SD}}(r)$ and shock front phase $\Theta_{\mathrm{SF}}(r)$, using one-dimensional Fourier transform of azimuthal profiles $I_{r}(\theta)$. For $m=2$ it is given

TABLE 2

Values Derived from Fourier Spectra

\begin{tabular}{cccccl}
\hline \hline \multirow{2}{*}{ Band } & $m$ & $\begin{array}{c}\text { Pitch Angle } \\
(\mathrm{deg})\end{array}$ & $\begin{array}{c}\text { Normalized } \\
\text { Amplitude }\end{array}$ & $\begin{array}{c}\text { Extent } \\
(\mathrm{pc})^{\mathrm{a}}\end{array}$ & \multicolumn{1}{c}{ Comment } \\
\hline$K_{s} \ldots \ldots \ldots \ldots \ldots \ldots$. & 2 & $83 \pm 7.1$ & 1.00 & 600 & Bar structure \\
$(V+R) \ldots \ldots \ldots \ldots$. & 1 & $76 \pm 14.0$ & 1.00 & 200 & Barlike \\
& 1 & $15 \pm 1.0$ & 0.50 & $\ldots$ & \\
& 2 & $24 \pm 1.2$ & 0.57 & 400 & Arms at short bar tips \\
& 2 & $-83 \pm 6.9$ & 0.58 & 300 & Short bar structure \\
& 2 & $76 \pm 6.9$ & 1.00 & 250 & Bar structure \\
& 2 & $-10 \pm 2.1$ & 0.41 & $300-450$ & Leading arms \\
\hline
\end{tabular}

a The extents of the modes are taken in radii from the galaxy nucleus. 


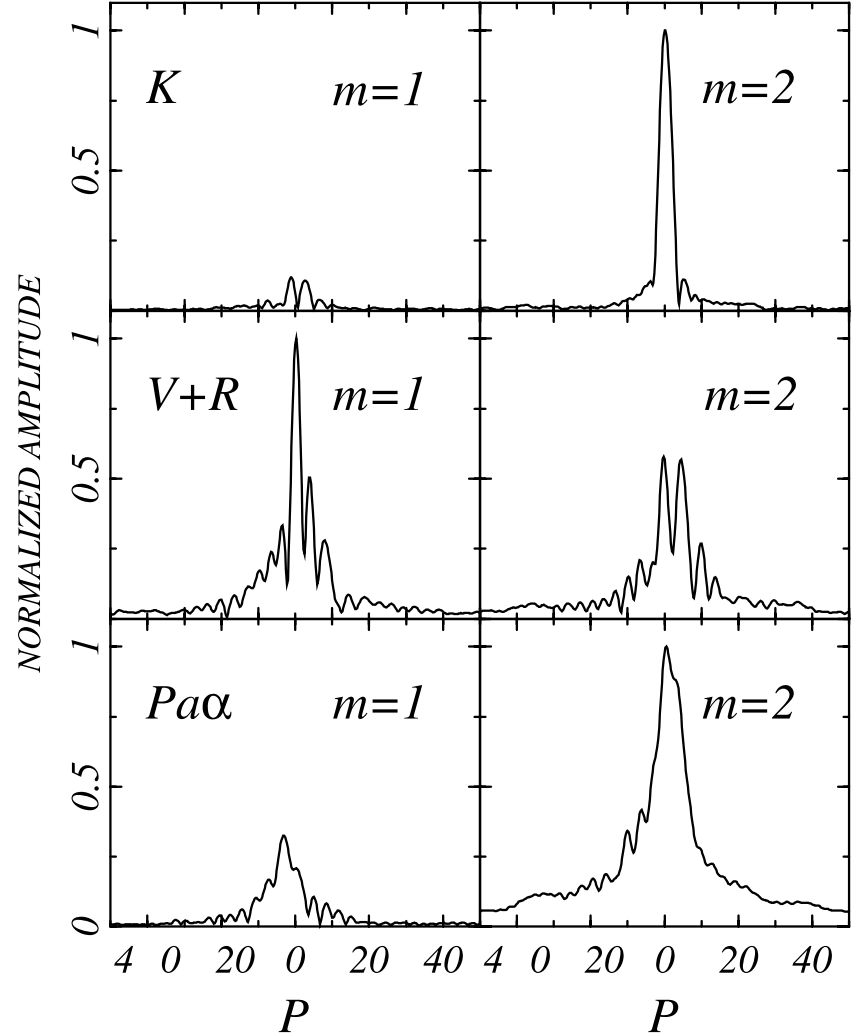

FIg. 5.-Two-dimensional Fourier spectra coefficients $A(p, m)$ of the inner $2 \mathrm{kpc}$ of NGC 1241. $P$ is related to the pitch angle $\alpha$ by $P=-m / \tan \alpha$. The $m=2$ mode is the sole component in the $K_{\mathrm{s}}$ band. The $(V+R)$ band is very complex in nature, with a predominant strong $m=1$ component and weaker leading plus trailing $m=2$ components. The barlike, $P=0$, and the leading arms, $P=-10$, structures are conspicuous in the $\mathrm{Pa} \alpha, m=2$ mode

by

$$
F_{2}(r)=\int_{-\pi}^{+\pi} I_{r}(\theta) e^{-2 i \pi} d \theta .
$$

The phase $\Theta(r)$ can be obtained from

$$
\Theta_{2}(r)=\tan ^{-1} \frac{\operatorname{Re}\left[F_{2}(r)\right]}{\operatorname{Im}\left[F_{2}(r)\right]},
$$

where Re and Im are the real and imaginary parts of the complex Fourier coefficients, respectively. The relative behavior of both phases is shown in Figure 2 of Puerari \& Dottori (1997) for trailing and leading S- and Z-type waves, and this method was also successfully used by VeraVillamizar et al. (2001) to find CR in barred galaxies. This procedure essentially consists of rectifying the azimuthal profiles using the method of Beckman \& Cepa (1990), but in our case we measure the phase difference using onedimensional FFT instead of measuring directly by hand as done by Beckman \& Cepa (1990).

Shock-induced star formation produces an azimuthal gradient of ages across the spiral arm that has opposite signs on either side of the CR. We assume here that the $\Theta_{\mathrm{SD}}(r)$ is represented by $\Theta_{K}(r)$, and $\Theta_{\mathrm{SF}}(r)$ by $\Theta_{(V+R)}(r)$ and $\Theta_{\mathrm{Pa} \alpha}(r)$, the latter representing the most recently formed stars, an azimuthal profile phase difference $\left[\Theta_{(V+R)}(r)-\Theta_{K}(r)\right]=0$, and/or $\left[\Theta_{K}(r)-\Theta_{\operatorname{Pa} \alpha}(r)\right]=0$, indicating the presence of a CR.

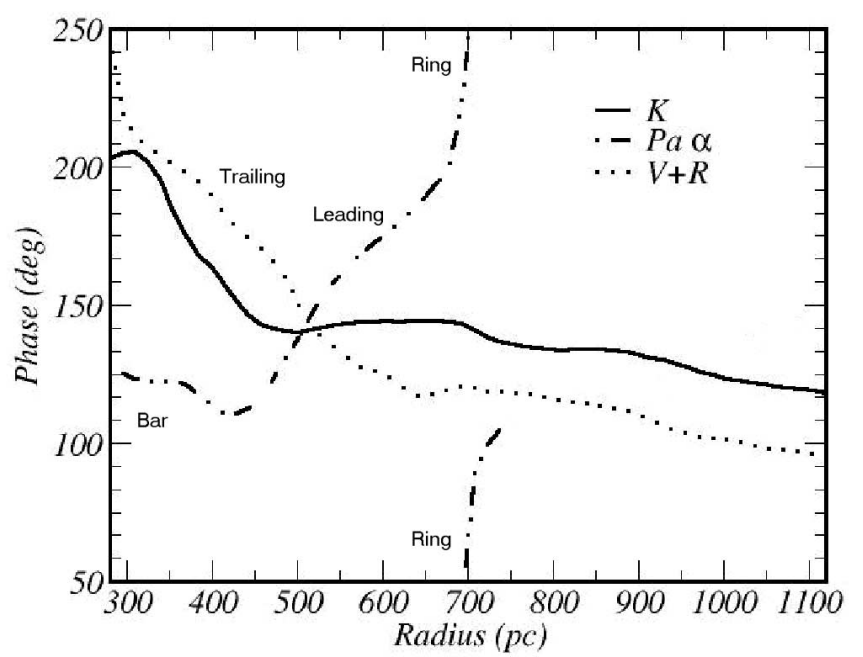

FIG. 6.-Azimuthal profile phase angle vs. radius calculated with onedimensional FFT. The corotation radius is $r \simeq 520 \mathrm{pc}$. The $\mathrm{Pa} \alpha$ plot shows the presence of a nuclear bar and a leading pattern with a pitch angle $i=29^{\circ}$.

We also searched for the existence of the $m=1$ component, the phase $\Theta(r)$ for this component being given by

$$
\Theta_{1}(r)=\tan ^{-1} \frac{\operatorname{Re}\left[F_{1}(r)\right]}{\operatorname{Im}\left[F_{1}(r)\right]}
$$

where

$$
F_{1}(r)=\int_{-\pi}^{+\pi} I_{r}(\theta) e^{-1 i \pi} d \theta .
$$

As shown in Figure 6, the general trends of the trailing $m=2$ modes $\Theta_{K}(r)$ and $\Theta_{(V+R)}(r)$ are similar, with a clear cutoff at $r \approx 520$ pc outside the estimated end of the minibar radius in the $K_{s}$ band. The $\Theta_{\mathrm{Pa} \alpha}(r)$ clearly shows the central $r \approx 250$ pc bar and the leading $m=2$ arm extending outward from the $\mathrm{Pa} \alpha$ bar, intercepting $\Theta_{K}(r)$ at the same point as $\Theta_{(V+R)}(r)$ at $r \approx 520 \mathrm{pc}$, which points to a connection between the youngest burst of star formation and the previous one. The fact that leading arms in differentially rotating disks tend to unwind rapidly suggests a very young age for this perturbation. Similar behavior has been found for bar-induced star formation in the Large Magellanic Cloud (Dottori et al. 1996). From the spectroscopic observations we derived the angular speed of the circumnuclear pattern in NGC 1241, $\Omega_{\mathrm{CN}}=350 \pm 50 \mathrm{~km} \mathrm{~s}^{-1} \mathrm{kpc}^{-1}$ (see 3.4). The phase difference between $\Theta_{K}(r)$ and $\Theta_{(V+R)}(r)$ amounts to $30^{\circ}$, much higher than phase differences found in disk largescale perturbations (Vera-Villamizar et al. 2001; Puerari \& Dottori 1997; Beckman \& Cepa 1990; Puerari et al. 2000), in agreement with the much higher angular velocity found at circumnuclear scales.

\subsection{The Connection between Circumnuclear and Global Scale Kinematics}

The central $5^{\prime \prime}$ region of NGC 1241 has a strong velocity gradient along the major axis, but the other slit positions showed no significant noncircular motions in this region, suggesting that the velocity gradient is mainly due to rotation. The radial velocity points along the southeast side of 


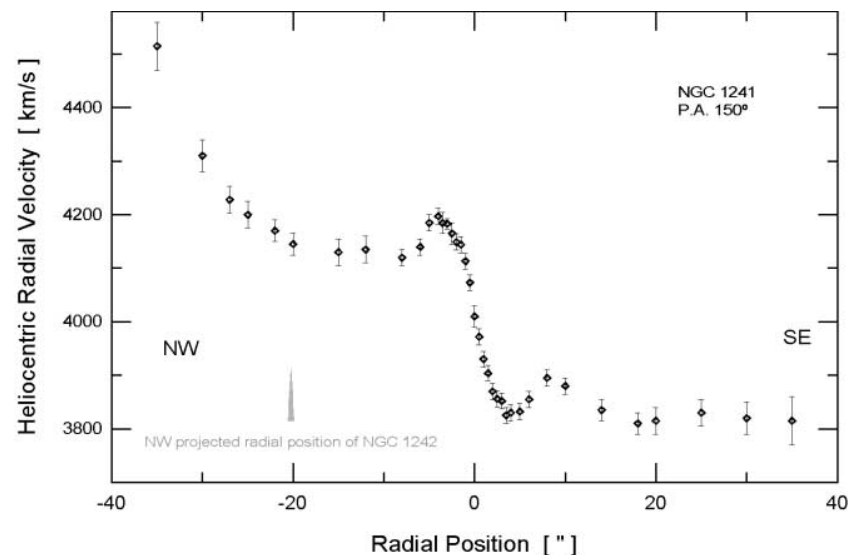

FIG. 7.-Radial velocities along P.A. $=150^{\circ}$. Note the marked differences between the southeast and northwest sides.

the major optical axis of the galaxy were used to derive the approximate global rotation curve (Fig. 7).

We derived an inclination of $52^{\circ}$ from the geometric parameters in the $(V+R)$ and $\left(J, K_{s}\right)$ images, which are consistent with those quoted by Tully (1988).

Fitting of Satoh's density distribution (see Binney \& Tremaine 1987) to the inner rotation curve shown in (Fig. 8) gave a mass of $\sim 1.5 \times 10^{10} M_{\odot}$ in the inner kiloparsec. Farther out, up to $r \sim 3.5 \mathrm{kpc}$, non-Keplerian motions begin to be evident in the rotation curve because the shape of the rotation curve in this region is strongly affected by the non-axisymmetric potential of the large-scale bar (e.g., Athanassoula 1992; Piner et al. 1995). The importance of the fact that the rotation curve fits Satoh's density law is that the parameters of this law lead naturally (but not exclusively) to the presence of a flat component in the matter distribution, consistent with the presence of a disk inside the spherical central kiloparsec. Satoh's density law also reproduces the "bump" found at the end of the so-called solid body part of the rotation curve, in contrast to the conventional fitting, which forces an inner solid body plus a flat outer part and does not reproduce the bump.

The unresolved nuclear emission lines $\mathrm{H} \alpha$ and $[\mathrm{N}$ II] $\lambda \lambda 6548,6584$ have line ratios typical of Seyfert 2 galaxies

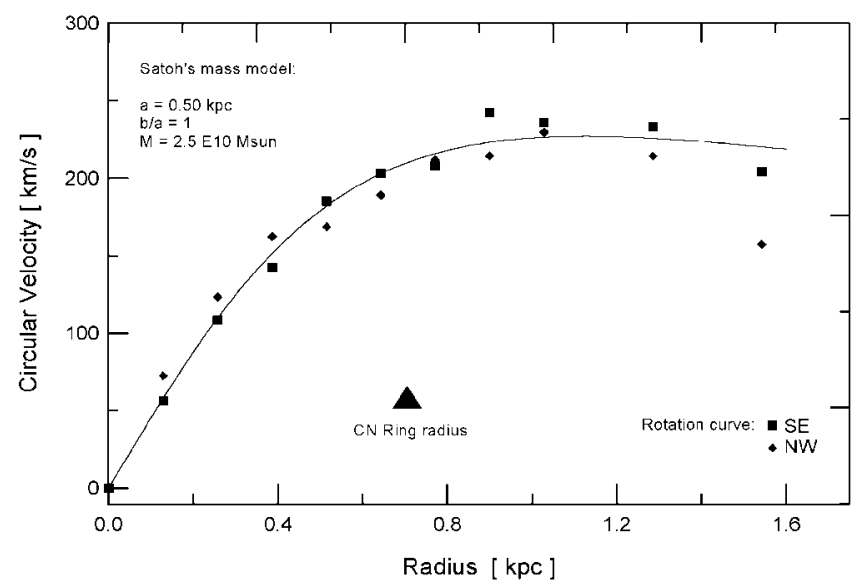

FIG. 8.-Satoh's disk model fitted to the inner rotation curve. Noncircular motions are evident beyond $1.5 \mathrm{kpc}$.

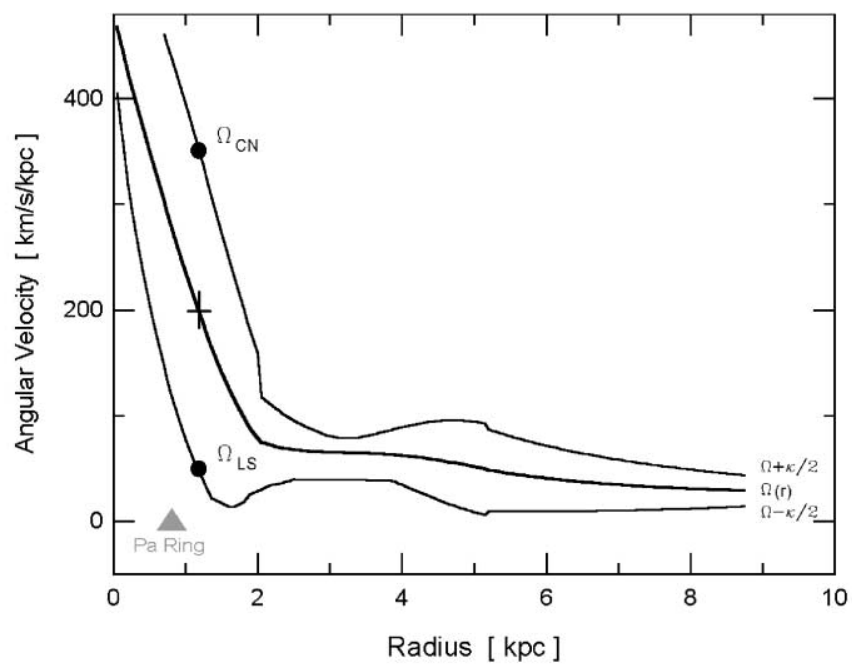

FIG. 9.-Angular velocity curve $\Omega(r)$, Lindblad curves $\Omega(r) \pm \kappa(r) / 2$, large-scale pattern angular speed $\Omega_{\mathrm{LS}}$, and circumnuclear pattern angular speed $\Omega_{\mathrm{CN}}$. Note the coincidence of the large-scale ILR [radial position of $\Omega_{\mathrm{LS}}$ on the curve $\Omega(r)-\kappa(r) / 2$ ] with the circumnuclear OLR [radial position of $\Omega_{\mathrm{CN}}$ on the curve $\Omega(r)+\kappa(r) / 2$ ]. The uncertainty in the angular velocity value at $1.1 \mathrm{kpc}$ is marked.

(Vaceli et al. 1997) and show a broadening ( $\langle$ FWHM $\rangle \sim$ $500 \mathrm{~km} \mathrm{~s}^{-1}$ ) characteristic of this type of active nucleus.

The dust lanes in the large-scale bar (WFPC2 image) correspond to the shocked regions (Prendergast 1962) of the galaxy and lie at the leading edge of the bar and the trailing edge of the global spiral arms beyond the end of the largescale bar, this configuration being consistent with the assumed direction of rotation because such a configuration means that the spiral arms in the outer disk are trailing. We used the derived rotation curve to calculate the angular velocity curve and the Lindblad curve $\Omega-\kappa / 2$ (Fig. 9), where $\kappa$ is the epicyclic frequency. In order to find the pattern speed, we made the normal assumption (Sparke \& Sellwood 1987) that the corotation lies at the end of the large-scale bar, $R_{\mathrm{bar}}=5.3 \pm 0.3 \mathrm{kpc}$ (Table 1$)$. We found $\Omega_{\mathrm{LS}} \sim 45 \pm 5 \mathrm{~km} \mathrm{~s}^{-1} \mathrm{kpc}^{-1}$ and that the circumnuclear ring of star formation ( $540 \mathrm{pc}<R_{\text {ring }}<900 \mathrm{pc}$ ) is located well inside the inner Lindblad resonance (ILR; $R_{\mathrm{ILR}}=1.2 \pm 0.1$ $\mathrm{kpc})$, but it was not possible to determine if there is a second ILR as suggested by numerical simulations (Piner et al. 1995). This is a key problem that needs to be solved in order to understand the mechanisms that govern the feeding of the accretion disk around the supermassive black hole postulated by the AGN canonical model and would require kinematics at subarcsecond resolution.

As pointed out by Friedli \& Martinet (1993), in order to minimize chaos, it would be expected that the end of the nuclear bar or its corotation resonance would match the ILR of the large-scale bar, but, in fact, the stellar nuclear bar ends and has its corotation well inside the ILR radius. This fact and the lack of evidence for a second ILR would point to the circumnuclear perturbation being dynamically decoupled from the large-scale perturbation. Nevertheless, a possible connection between circumnuclear and global dynamics is that the circumnuclear outer Lindblad resonance (OLR), at a radius of approximately $R_{\mathrm{CN}-\mathrm{OLR}}=$ $1.2 \pm 0.2 \mathrm{kpc}$, coincides (within the uncertainties inherent in this type of study) with the large-scale ILR (see Table 3). 
TABLE 3

Dynamical Radil Derived for NGC 1241

\begin{tabular}{|c|c|c|}
\hline Feature & $\begin{array}{c}\text { Radius } \\
\text { (kpc) }\end{array}$ & Comment \\
\hline Tidal radius ${ }^{\mathrm{a}}$ & 41 & NGC 1242 as point mass \\
\hline Outer Lindblad resonance & $16.7 \pm 1.5$ & End of main spiral pattern \\
\hline Corotation resonance & $5.3 \pm 0.5$ & $\Omega_{P}=45 \pm 5 \mathrm{~km} \mathrm{~s}^{-1} \mathrm{kpc}^{-1} ;$ morphological \\
\hline Inner Lindblad resonance & $1.2 \pm 0.1$ & \\
\hline Circumnuclear outer Lindblad resonance ............... & $1.2 \pm 0.1$ & \\
\hline Circumnuclear corotation resonance.......................... & $0.52 \pm 0.05$ & $\Omega_{p}=350 \pm 50 \mathrm{~km} \mathrm{~s}^{-1} \mathrm{kpc}^{-1} ;$ morphological \\
\hline
\end{tabular}

${ }^{a}$ Using the estimated total masses and projected distance to NGC 1242 nucleus.

The dynamics described in the previous paragraph is enriched by the presence of the perturbing companion NGC 1242 , which appears projected at about $25 \mathrm{kpc}$ near the far north-northeast side (the most perturbed one) of NGC 1241. Moreover, the major-axis position angle and the radial intensity profiles seen in the Digitized Sky Survey (DSS) images change significantly beyond $0.57 R_{\mathrm{gal}}$. Ellipse fitting of the circumnuclear ring infrared images shows that the ring is more perturbed at its north-northeast side, while our data show that the north-northwest side of the rotation curve is perturbed. This is consistent with the idea suggested by several authors (see, e.g., Dahari 1984 for a complete discussion) that the perturbation of a companion galaxy modifies the dynamics and morphology of the galactic systems from global scales down to the nuclear region, allowing an enhanced accretion rate at the very center of the galaxy. Recent numerical simulations of McLeish's interacting galaxy, using the TREESPH code of Hernquist \& Katz (1989), have shown (Díaz et al. 2000) that the rotation curve of this giant spiral galaxy may be asymmetrically perturbed by a near flyby dwarf galaxy with a $1: 1$ resonant orbit, an important part of the disk gas being driven to the central region of the galaxy. A similar analysis of the interacting pair NGC 1241/NGC 1242 would help to quantify the importance of NGC 1242 in driving gas to the central part of NGC 1241.

\subsection{Inner Pattern Origin and Age}

The mass of cold molecular gas in the central regions of NGC 1241 can be estimated from ${ }^{12} \mathrm{CO}(1-0)$ luminosity (Maiolino et al. 1997) obtained by an unresolved singlepoint observation with beam centered on the galactic nucleus to give the $\mathrm{CO}$ luminosity inside $r=7.2 \mathrm{kpc}$. Considering the beam coupling efficiency and the uncertainties in telescope efficiency and drifts in the calibration claimed by Maiolino et al. (1997), we estimate $L_{\mathrm{CO}}=(6.0 \pm 1.2) \times$ $10^{4} L_{\odot}$, which gives a mass of molecular hydrogen $M\left(\mathrm{H}_{2}\right)$ $=(6 \pm 1) \times 10^{9} \quad M_{\odot} \quad\left(\right.$ using $M\left(\mathrm{H}_{2}\right)=9.2 \times 10^{4} L_{\mathrm{CO}}\left(L_{\odot}\right)$ $\left[M_{\odot}\right]$; Kenney \& Young 1989). This represents an amount of molecular gas larger than that found in $90 \%$ of field spiral galaxies (Heckman et al. 1989). In most of the objects small amounts of molecular gas are found beyond $6 \mathrm{kpc}$ (Maiolino et al. 2000), and if we assume that most of this gas is arranged in an exponential disk of circumnuclear scale length, the inner kiloparsec would harbor at least $36 \%$ of the total mass of $\mathrm{H}_{2}$, or about $\sim 2 \times 10^{9} M_{\odot}$. Based on these assumptions, the mass of $\mathrm{H}_{2}$ would be a large fraction $(\geq 13 \%)$ of the dynamical mass limit inside $1 \mathrm{kpc}$, derived from the rotation curve. This is about half the gas fraction found in the central kiloparsec of the major merger galaxy
NGC 7252 (Schweizer 1982) and is similar to that occurring in NGC 2782 (Jogee, Kenney, \& Smith 1999). In the next paragraph we discuss the possible connection between large- and small-scale perturbations that may act in NGC 1241 if the calculated amount of gas is indeed concentrated in the central region, as usually observed in spiral galaxies. Although this argument may be somewhat attenuated by the coarseness of the radio observation, it may induce radio astronomers to refine their $\mathrm{CO}$ observations of this interesting galaxy.

Several models have been proposed to account for the origin of nuclear gaseous bars when such a large amount of gas is available, models that locally generate a spiral pattern in a self-gravitating circumnuclear disk seeming to be the more appropriated (Heller \& Shlosman 1994; Combes 1994; Friedli \& Martinet 1993). In such models the inner pattern speed $\Omega_{\mathrm{CN}}$ would be gravitationally decoupled from the large-scale stellar bar pattern speed $\Omega_{\mathrm{LS}}$, as well as from the induced large-scale gas response. The models predict that $\Omega_{\mathrm{CN}} \gg \Omega_{\mathrm{LS}}$, which was in fact observed by us in NGC 1241 (see $\S \S 3.3$ and 3.4). A scenario in which the circumnuclear bar is directly induced by the large-scale stellar bar perturbation is precluded, since the inner bar is well inside the outer pattern ILR, which cannot be transposed by the stellar density wave. The large-scale stellar bar might induce a perturbation in the gaseous phase, which in turn might indeed migrate inward through the ILR, but lack of penetration of the outer dust lanes into the nuclear region precludes such a secondary mechanism (Englmaier $\&$ Shlosman 2000), unless we are witnessing the postdecoupling era, in which case angular velocity patterns should be $\Omega_{\mathrm{CN}} \ll \Omega_{\mathrm{LS}}$ (Heller, Shlosman, \& Englmaier 2001), contrary to what we observed in this galaxy.

We could not make an estimate of the age of a locally generated circumnuclear pattern through presently existing models because it would have required high spatial resolution two-dimensional spectroscopy in order to derive inflow rates, subarcsecond mass distribution, etc. Instead we used the models of Junqueira \& Combes (1996), who numerically modeled galactic disks with stellar and gaseous components to study the evolution over time of a perturbation born in a stellar disk and its coupling and propagation to the gaseous counterpart. Junqueira \& Combes (1996) analyzed the relative behavior of the Fourier radial density function $S_{m}(r)$ (see $\S 3.2$ ) of stars and gas over periods spanning between 20 and $2000 \mathrm{Myr}$. A qualitative general trend in these models is that the stellar perturbation is dominant in the perturbed gas early in the development of perturbation. Later on the stellar perturbation progressively dispels and the radial density function of the perturbed gas becomes relatively more 


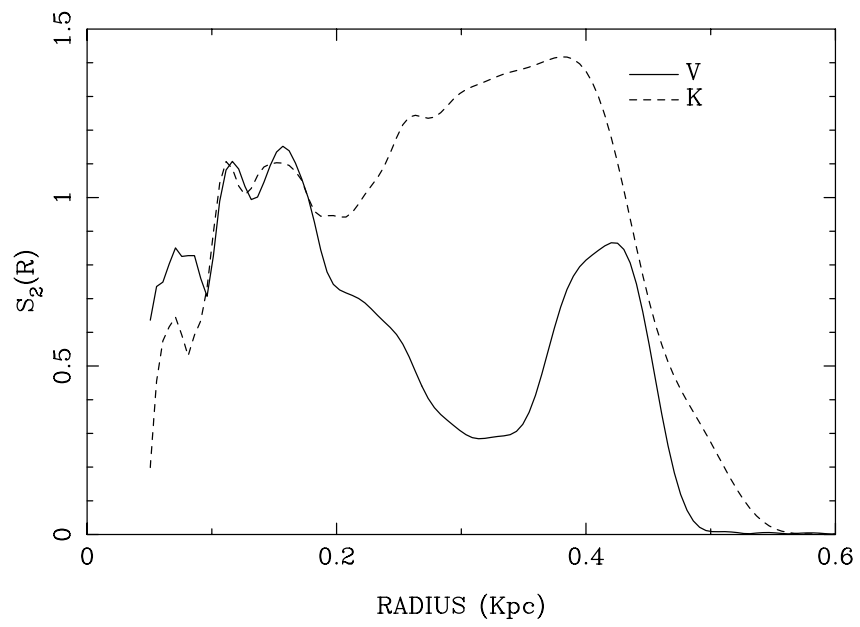

Fig. 10.-Fourier radial density function for $m=2, S_{2}(r)$, in $K_{s}$ (old stellar population) and $(V+R)$ (newly formed stars). Note the predominance of the old stellar population $S_{2}(r)$ on that of the newly formed stars.

important. Vera-Villamizar et al. (2001) used the relative behavior of $S_{m}(r)$ for the old stellar disk and newly formed stars as an indicator of age, and when we made this comparison, we saw that the $S_{m}(r)$ in $(V+R)$ and $\mathrm{Pa} \alpha$ images was stronger than that in $K_{s}$ images (see Fig. 10), pointing to the central region perturbation of NGC 1241 as being very young with an age of only a few tens of million years. Even so, the high angular pattern speed $\left(\Omega_{\mathrm{CN}}=350 \pm 50 \mathrm{~km} \mathrm{~s}^{-1}\right.$ $\left.\mathrm{kpc}^{-1} ; \S 3.4\right)$ in the central region means that it has made between five and 10 revolutions since its formation. Caution is necessary in using the model of Junqueira \& Combes (1996) because it is more suitable for disk radial scales of about $\approx 5 \mathrm{kpc}$, and more specific models with small-scale grids need to be developed to accurately confirm the lifetime of the perinuclear perturbation.

\section{DISCUSSION AND FINAL REMARKS}

In this paper we use $K, J, \mathrm{~Pa} \alpha$, and $(V+R)$ images and 1."5 resolution spectroscopy to analyze the central kiloparsec of galaxy NGC 1241, identifying structures by means of their photometric colors and the use of two- and onedimensional Fourier techniques.

We found that the complexity of the spiral pattern increases from the NIR to the visual, the $K$-band pattern being purely bimodal while the $(V+R)$ band pattern needed many Fourier components to explain the structure of the arms. This is in agreement with what is known for all large-scale arm patterns, namely, that the arms appear much simpler in NIR than in optical bands and that the classification of spirals is very different when NIR bands are used instead of shorter wavelength bands. In turn, it also indicates that it is not necessary to call on a different perturbation than the traditional spiral density wave acting on the old stellar population of the galaxy disk as an origin for the 100 pc scale arm pattern.

$\mathrm{Pa} \alpha$-band images revealed the presence of an emitting ring (Böker et al. 1999) embracing a barlike structure and a couple of faint leading arms. If the absorption had not been so strong, the ring would have been visible at $(V+R)$ images because of the leak of $\mathrm{H} \alpha$ photons in the $R$ band.
Purely gaseous bars can be triggered by self-gravitational instabilities in circumnuclear environments with large amounts of molecular gas and with strong velocity gradients, although an emission bar can also be induced by the small stellar bar observed in the optical and NIR broad bands by causing Jeans instabilities in the gathered gas. In the case of NGC 1241 the bar would not be accompanied by dust lanes as proposed by some authors, which might imply that the fraction of nuclear bars in AGNs, as well as their participation in AGN feeding, is larger than that suggested by authors such as Laine et al. (2002).

The angular speed of the inner pattern is very high because of the high rotational velocity of the inner regions. The most probable scenario for the circumnuclear secondary bar plus spiral pattern is that of an inner perturbation kinematically decoupled from the large-scale pattern, according to Englmaier \& Shlosman (2000) and Heller et al. (2001).

The corotation resonance does match the end of the inner bar and is well inside the radius of the circumnuclear ring. Numerical simulations of inner pattern formation via self-gravitational instability (e.g., Heller \& Shlosman 1994) suggest that the best arrangement corresponds to the corotation radius of the nuclear bar being approximately equal to the radius of the ILR of the primary bar, reducing the fraction of the chaotic orbits (Pfenniger \& Norman 1990). In the case of NGC 1241 the inner pattern OLR matches the global pattern ILR, within the uncertainties of the calculations, although, because the domains of both resonances overlap, the observed situation could be just a coincidence. Nevertheless, there is growing support for theoretical scenarios in which one of the inner pattern resonances is coincident with the large-scale pattern ILR. However, the recently published work of Regan \& Teuben (2003), indicating that barred galaxies lack an ILR feature, would preclude this mechanism in the case of NGC 1241, and it may be the transition of the gas to $x 2$ orbits that causes the nuclear ring. In order to further understand the origin and stability of the circumnuclear structures of NGC 1241 and similar galaxies, the work described in this paper could be complemented by using high-resolution NIR two-dimensional spectra to investigate the peculiar motions of the small-scale structures revealed in this study and to model them in greater detail.

The Gemini $8 \mathrm{~m}$ telescopes are an international partnership managed by the Association of Universities for Research in Astronomy (AURA), Inc., under a cooperative agreement with the National Science Foundation (NSF). The NASA/ESA Hubble Space Telescope is operated by AURA, Inc., under NASA contract NAS 5-26555. We thank M. P. Agüero for fitting the components in the brightness radial profiles. We are indebted to B. Garcia-Lorenzo for introducing the preliminary version of this work at the workshop held in La Palma in 2001 May. H. D. thanks the Brazilian institutions Conselho Nacional de Pesquisas (CNPq) and Coordenadora para Aperfeiçoamento do Pessoal de Ensino Superior (CAPES). This research is also partially supported by Brazilian grants MEGALIT/ Millennium and PRONEX and the Argentinean Consejo Nacional de Investigaciones Científicas y Técnicas (CONICET) and Agencia Córdoba Ciencia (ACC). 


\section{REFERENCES}

Athanassoula, E. 1992, MNRAS, 259, 345

Beckman, J. E., \& Cepa, J. 1990, A\&A, 229, 37

Binney, J., \& Tremaine, S. 1987, Galactic Dynamics (Princeton: Princeton Univ. Press)

Block, D. L., Bertin, G., Stockton, A., Grosbøl, P., Moorwood, A. F. M., \& Peletier, R. F. 1994, A\&A, 288, 365

Block, D. L., \& Puerari, I. 1999, A\&A, 342, 627

Böker, T., et al. 1999, ApJS, 124, 95

Buta, R., \& Combes, F. 1996, Fundam. Cosmic Phys., 17, 95

Buta, R., Treuthardt, G., Byrd, G., \& Crocker, D. 2000, AJ, 120, 1289

Combes, F. 1994, in Mass Transfer Induced Activity in Galaxies, ed.

I. Shlosman (Cambridge: Cambridge Univ. Press), 170

Considère, S., \& Athanassoula, E. 1982, A\&A, 111, 28

Cunow, B. 2001, MNRAS, 323, 130

Dahari, O. 1984, AJ, 89, 966

Díaz, R. J., Carranza, G., Dottori, H., \& Goldes, G. 1999, ApJ, 512, 623

Díaz, R. J., Rodrigues, I., Dottori, H., \& Carranza, G. 2000, AJ, 119, 111

Dottori, H. 1990, in ESO/CTIO Workshop on Bulges of Galaxies, ed.

B. J. Jarvis \& D. M. Terndrup (Paris: ESO), 299

Dottori, H., Bica, E., Clariá, J. J., \& Puerari, I. 1996, ApJ, 461, 742

Elmegreen, B. G., Elmegreen, D. M., \& Montenegro, L. 1992, ApJS, 79, 37

Englmaier, P., \& Shlosman, I. 2000, ApJ, 528, 677

Friedli, D., \& Martinet, L. 1993, A\&A, 277, 27

Heckman, T., Blitz, L., Wilson, A., Armus, L., \& Miley, G. 1989, ApJ, 342, 735

Heller, C. H., \& Shlosman, I. 1994, ApJ, 424, 84

Heller, C. H., Shlosman, I., \& Englmaier, P. 2001, ApJ, 553, 661

Hernquist, L., \& Katz, N. 1989, ApJS, 70, 419

Ivanov, V. D., Rieke, G. H., Groppi, C. E., Alonso-Herrero, A., Rieke, M. J., \& Engelbracht, C. W. 2000, ApJ, 545, 190

Jogee, S., Kenney, J., \& Smith, B. J. 1999, ApJ, 526, 665

Junqueira, S., \& Combes, F. 1996, A\&A, 312, 703

Kalnajs, A. J. 1975, in La Dynamique des Galaxies Spirales, ed. L. Weliachew (Paris: CNRS), 103

Kenney, J., \& Young, J. 1989, ApJ, 344, 171

Knapen, J. H., Beckman, J. E., Shlosman, I., Peletier, R. F., Heller, C. H., \& de Jong, R. S. 1995, ApJ, 443, L73
Laine, S., Shlosman, I., Knapen, J., \& Peletier, R. 2002, ApJ, 567, 97

Lindblad, P. A. B., Lindblad, P. O., \& Athanassoula, E. 1996, A\&A, 313, 65

Maiolino, R., Alonso-Herrero, A., Anders, S., Quillen, A., Rieke, M., \& Tacconi-Garman, L. 2000, ApJ, 531, 219

Maiolino, R., Ruiz, M., Rieke, M., \& Papadopoulus, P. 1997, ApJ, 485, 552

Martini, P., \& Pogge, R. 1999, AJ, 118, 2646

Pastoriza, M., Dottori, H., Terlevich, E., Terlevich, R., \& Diaz, A. 1993, MNRAS, 260, 177

Persic, M., \& Salucci, P. 1995, ApJS, 99, 501

Pfenniger, D., \& Norman, C. 1990, ApJ, 363, 391

Piner, B. G., Stone, J. M., \& Teuben, P. J. 1995, ApJ, 449, 508

Prendergast, K. H. 1962, in Interstellar Matter in Galaxies, ed. L. Woltjer (New York: W. A. Benjamin Inc.), 217

Puerari, I., Block, D. L., Elmegreen, B. G., Frogel, J. A., \& Eskridge, P. B. 2000, A\&A, 359, 932

Puerari, I., \& Dottori, H. 1992, A\&AS, 93, 469 1997, ApJ, 476, L73

Regan, M. W., \& Mulchaey, J. S. 1999, AJ, 117, 2676

Regan, M. W., \& Teuben, P. J. 2003, ApJ, 582, 723

Schmitt, H., Storchi-Bergmann, T., \& Fernandes, R. C. 1999, MNRAS, 303, 173

Schweizer, F. 1976, ApJS, 31, 313 .1982, ApJ, 252, 455

Seigar, M. S., \& James, P. A. 1998, MNRAS, 299, 685

Shlosman, I., Frank, J., \& Begelman, M. C. 1989, Nature, 338, 45

Sparke, L., \& Sellwood, J. 1987, MNRAS, 225, 653

Terlevich, R., \& Melnick, J. 1985, MNRAS, 213, 841

Tully, R. B. 1988, Nearby Galaxies Catalog (Cambridge: Cambridge Univ. Press)

Vaceli, M. S., Viegas, S. M., Gruenwald, R., \& de Souza, R. E. 1997, AJ, 114,1345

Vera-Villamizar, N., Dottori, H., Puerari, I., \& de Carvalho, R. 2001, ApJ, 547,187 